\title{
Numerical Examination of Photon Recycling as an Explanation of Observed Carrier Lifetime in Direct Bandgap Materials
}

\author{
JOSEPH W. PARKS JR. ${ }^{a}$, KEVIN F. BRENNAN $^{\mathrm{a}, *}$ and ARLYNN W. SMITH ${ }^{\mathrm{b}}$ \\ ${ }^{a}$ Microelectronics Research Center, Georgia Institute of Technology, Atlanta, GA 30332; \\ ${ }^{\mathrm{b}}$ ITT Night Vision, Roanoke, VA 24019
}

\begin{abstract}
Photon recycling is examined as an explanation for the observed large carrier lifetimes in an InP/InGaAs photodiode. This effect extends the effective carrier lifetime within a device by re-absorbing a fraction of the photons generated through radiative band-toband recombination events. In order to predict the behavior of this carrier generation, photon recycling has been added to our two-dimensional macroscopic device simulator, STEBS-2D. A ray-tracing preprocessing step is used to map all of the possible trajectories and absorption of various wavelengths of emitted light from each originating node within the device. The macroscopic simulator uses these data to determine the spatial location of the re-absorbed radiation within the geometry of the device. By incorporating the ray tracer results with the total quantity and spectral content of recombined carriers at each node within the simulation, the recycled generation rate can be obtained. A practical application of this model is presented where the effects of photon recycling are used as a possible explanation of the discrepancy between the theoretically predicted and experimentally observed radiative recombination rate in a double heterostructure photodetector.
\end{abstract}

Keywords: Photon recycling, reabsorption, self-excitation, macroscopic simulation

\section{INTRODUCTION}

It is often observed that radiative band-to-band transitions are the dominant recombination mechanism in many high quality direct gap semiconductors. Those carriers which recombine radiatively conserve energy through the liberation of a photon with a characteristic energy near the semiconductor bandgap. Photon recycling is the reabsorption of this emitted light elsewhere in the device to produce additional electron-hole pairs. Thus, photon recycling can be described as an additional generation mechanism which is tightly coupled to the total amount of radiative recombination over the range of the device. The effectiveness of photon recycling depends on many factors such as the dominance of

* Corresponding author. 
the radiative recombination mechanism and the light confinement properties of the structure.

The influence of photon recycling on the performance of many devices has been investigated [1 3], and in several cases it has been shown that neglecting this effect leads to improper analysis of the carrier transport. Therefore, a general model including the influence of photon recycling should be included within advanced macroscopic device simulators. Unfortunately, many of the models used to analyze photon recycling have relied on either one-dimensional or analytical approximations to estimate the distribution of the generated carriers, and general multi-dimensional models don't exist.

The primary impetus behind this study is to develop a fully numerical, general two-dimensional photon recycling model which can be directly coupled into the STEBS-2D [4] macroscopic device simulator. To achieve the desired result, ray tracing techniques are employed to monitor the trajectories and absorption of light propagating through the device geometry. Since the optical properties of the devices of interest are spatially inhomogeneous, and since the generated light from the recombination events has a non-uniform spectral content, the ray tracer must be used to map the absorption of light originating from any point in the device over a range of wavelengths.

As an example of the utility of the model, an InP/InGaAs double heterostructure photodiode [5] is examined to determine whether the photon recycling phenomenon can be used to explain the discrepancy between theoretically predicted and experimental lifetimes. Experimentally, the lifetime is measured by monitoring the magnitude of the collected current as a light source is moved across the top of the device. Clearly from the geometry of the structure and the nature of the experiment, one-dimensional or analytical models are insufficient and a two-dimensional analysis is necessary.

\section{NUMERICAL MODEL}

The overall goal in the implementation of photon recycling is the mapping of the emission of photons at one point to their absorption at all other points in the simulation domain. This requires a discrete approach at the positional as well as the spectrum level. The spatial discretization of the simulation domain follows that of the electrical model, while the continuum of emitted wavelengths is broken into several discrete energy ranges representative of the wavelengths which could originate from a given material.

Ray tracing techniques are used here to model the propagation of light through the crystal. A general purpose, three dimensional, ray tracing algorithm based on the idea of constrained volumes is used to provide the mapping of light emission to absorption [6]. The emitted rays are tracked from volume to volume, and the distance through each is used to determine the total absorption at each node. As the rays move between volumes of differing material, they are refracted and secondary reflected rays are generated. Implicit in this model are features such as wavelength dependent refraction, critical angle calculation, wavelength and angle dependent transmission coefficients, and various surface reflection models including antireflection coatings.

The ray tracing itself is fairly straight forward. From each node, a large number of rays are emitted in random directions. The path of each ray is followed until either the ray is attenuated or is coupled out of the device without possibility of return. For each initiating point, the detailed absorption within the device is catalogued. This process must be repeated for each simulated wavelength owing to the differing absorption and refraction indices. These data are arranged as a set of cross-referenced lists relating the initiating point of emission, the points contributing to the absorption, and the amount of attenuation.

The information from the ray tracing is incorporated into the source term of the current continuity equations of the macroscopic simulator as seen in the flowchart given in Figure 1. Within each iteration, the spectrum of the spontaneously emitted light is obtained for each node, $(x, y)$. The energy dependent spontaneous emission rate, $R$, is estimated through the use of the van-Roosbroeck Shockley relation given by Eq. (1) 


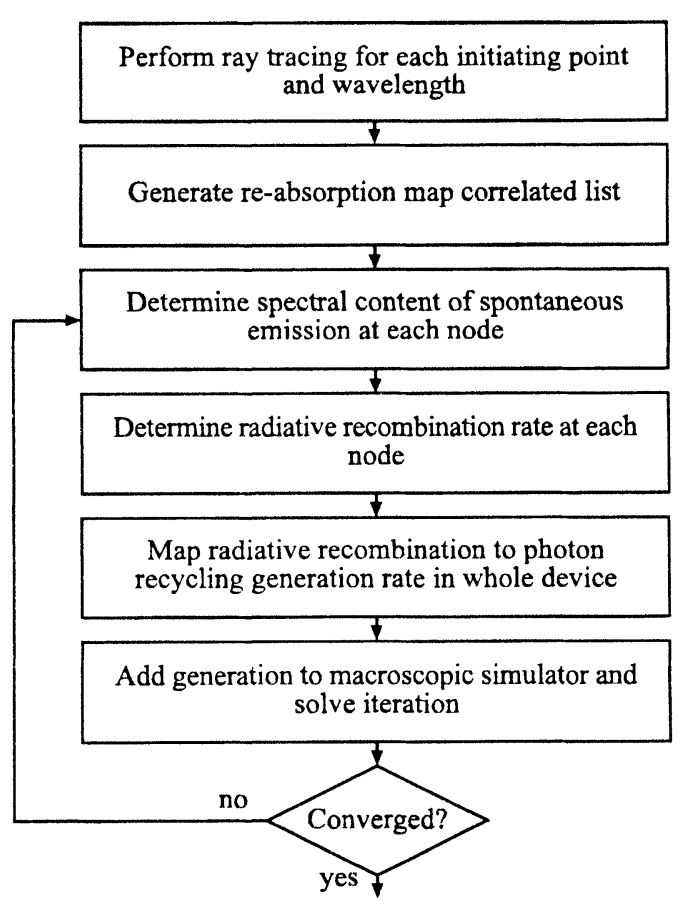

FIGURE 1 Flowchart depicting steps within the applied photon recycling model.

$$
R_{(x, y)}(v) d v=\frac{8 \pi(h v)^{2} n(v)^{2} \alpha(v)}{h^{2} c^{2}\left[\exp \left(\frac{h v-\Delta E_{f(x, y)}}{k T}\right)-1\right]}
$$

where $\Delta E_{f}$ is the current estimate of the difference between the electron and hole quasi-Fermi levels, and $\alpha$ and $n$ are the wavelength dependent absorption and index of refraction [7]. Next, an estimate of the total radiative recombination rate at the node is determined by:

$$
R_{\mathrm{RAD}(x, y)}=B\left(n_{(x, y)} p_{(x, y)}-n_{i}^{2}\right)
$$

where the radiative constant $B$ is taken to be the integral of Eq. (1) divided by $n_{i}^{2}$. The normalized spectrum produced from Eq. (1) is divided into non-overlapping energy ranges centered around the discrete wavelengths simulated by the ray tracer. The fraction, $f_{(x, y, \lambda)}$, of the overall spontaneous rate for each wavelength is taken to be the integral of Eq. (1) over each energy range normalized to the integral of the total spectrum. Using the output from the ray tracer, the total photon recycling generation rate at any point, $(l, k)$ can be determined by:

$$
G_{(l, k)}=\sum_{x, y}^{\Omega} R_{\mathrm{RAD}(x, y)} \frac{V_{(x, y)}}{V_{(l, k)}}\left[\sum_{\lambda} f_{(x, y, \lambda)} r_{(x, y, l, k, \lambda)}\right]
$$

where $r$ is the ray tracer results describing the fraction of the absorbed power at $(l, k)$ due to emission at $(x, y)$ for wavelength $\lambda$ and $V_{(x, y)}$ and $V_{(l, k)}$ are the control volume areas for the originating and absorbing elements.

The macroscopic model used in this study is the drift-diffusion subset of the hydrodynamic simulator. Here, Poisson's equation is coupled to the current continuity equations for inhomogeneous materials [4]. By including the generation rate from. Eq. (3) into the continuity equations and iterating until a self consistent solution is obtained, the effects from photon recycling may be fully included into the device simulation.

\section{SIMULATION RESULTS}

The aforementioned model, included in the macroscopic device simulator, is used to examine the affect of photon recycling upon the performance of the device sketched in Figure 2. For this device, excess carriers are produced from a 1.3 micron monochromatic light source ten microns in diameter moved laterally across the top surface. This light is absorbed exclusively in the InGaAs with an internal quantum efficiency of 97 percent. For the device in question, the measured minority carrier lifetime is approximately three times larger than that predicted from the theoretical radiative recombination rate. Photon recycling is conjectured here to be a possible cause of this discrepancy.

The two-dimensional device simulator cannot accurately capture the inherent three-dimensional nature of the diffusion of excess carriers in the original experiment. Therefore an assumption 


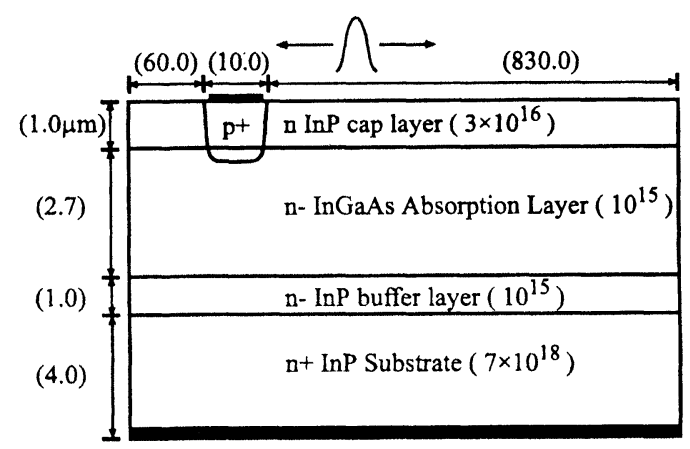

FIGURE 2 Illustration of device geometry and doping profiles for the photodiode under investigation. The device is nominally reverse biased to five volts and carrier excitation is through photogeneration from the laterally moving light source. All dimensions are in microns and doping in $\mathrm{cm}^{-3}$.

must be made upon the value of the non-simulated third dimension of the device. The calculated output current is first made to agree with the reported results using the experimentally obtained lifetime by adjusting the level of carrier injection by an appropriate choice of length for the third dimension. Once this control is established, the simulation is again performed using the lower, theoretically predicted, lifetime. The results of this simulation are shown by the dashed curve in Figure 3 for two different light intensities. Comparison of the experimental results to the calculations made neglecting photon recycling with the theoretical lifetime clearly show that the output current is greatly underestimated. The simulation is also performed with the inclusion of photon recycling. The curve marked with the solid squares in Figure 3 shows the calculated photocurrent including photon recycling assuming the theoretical carrier lifetime. Comparison of the experimental results to the calculations including photon recycling shows that the inclusion of the additional photon recycling generation rate increases the magnitude of the collected current to a value clearly resembling that of experiment. From these results, it is observed that photon recycling provides the mechanism to describe the disparity

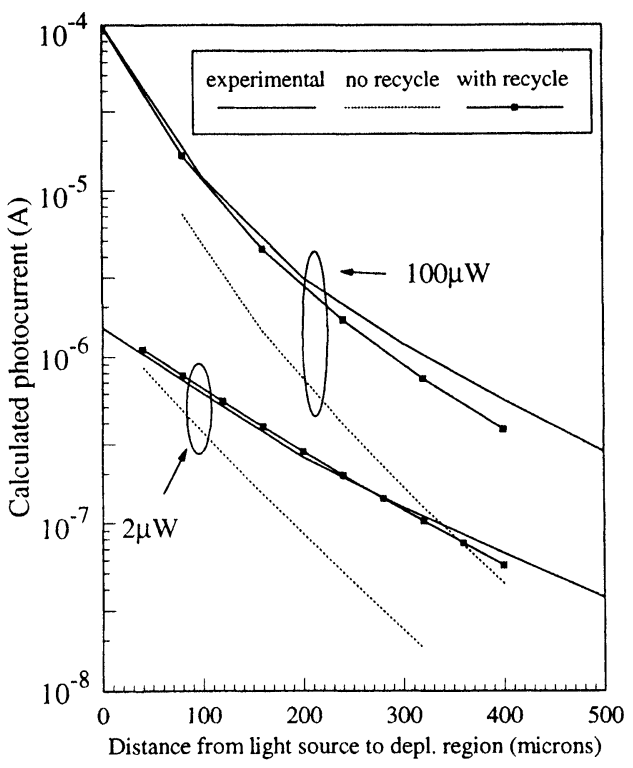

FIGURE 3 Calculated and experimental collection currents for the double heterostructure photodiode under $2 \mu \mathrm{W}$ and 100 $\mu \mathrm{W}$ incident optical power. Each case illustrates the calculated current both with and without the inclusion of photon recycling.

between the experimental and theoretical lifetimes observed in this device.

\section{CONCLUSIONS}

This paper describes a general purpose model used to include the effects of photon recycling into a macroscopic device simulator. A three-dimensional ray tracer is used to predict how spontaneously emitted light propagates and is absorbed within a semiconductor device. These data are then coupled to a two-dimensional macroscopic device simulator and are used in conjunction with the quantity and spectral content of the radiative recombination rate to predict the total carrier generation due to photon recycling. As an example of the model, the effect of photon recycling is examined in a double heterostructure InP/InGaAs photodiode where it is observed that the inclusion of photon recycling within the InGaAs restores the 
agreement between the experimentally and theoretically obtained values of carrier lifetime.

\section{References}

[1] Durbin, S. M. and Gray, J. L., "Numerical Modeling of Photon Recycling in Solar Cells", IEEE Trans. Elec. Dev., 41(2), pp. 239-245, Feb. 1994.

[2] Asbeck, P., "Self-Absorption Effects on the Radiative Lifetime in GaAs-GaAlAs Double Heterostructures", $J$. Appl. Phys., 48(2), pp. 820-822, Feb. 1977.

[3] Sieg, R. M. and Ringel, S. A., "Reabsorption, Band-gap Narrowing, and the Reconciliation of Photoluminescence Spectra with Electrical Measurements for Epitaxial n-InP", J. Appl. Phys., 80(1), pp. 448-458, July. 1996.

[4] Parks, J. W., Smith, K. F., Brennan, K. F. and Tarof, L. E., "Theoretical Study of Device Sensitivity and Gain Saturation of Separate Absorption, Grading, Charge and Multiplication InP/InGaAs Avalanche Photodiode", IEEE Trans. Elec. Dev., 43(12), pp. 2113-2121, Dec. 1996.

[5] Gallant, M. and Zemel, A., "Long Minority Hole Diffusion Length and Evidence for Bulk Radiative Recombination Lifetime in InP/InGaAs Double Heterostructures", Appl. Phys. Lett., 52(20), pp. 1686-1688, May 1988.

[6] Smith, A. W., Rohatgi, A. and Neel, S. C. (1990). "TEXTURE: A Ray Tracing Program for the Photovoltaic Community", Proc. $21^{\text {st }}$ IEEE Photovoltaic Spec. Conference.

[7] Pankove, J. I. (1971). Optical Processes in Semiconductors, Prentice-Hall, Englewood Cliffs, N. J.

\section{Authors Biographies}

Joseph W. Parks Jr. was born in Oak Ridge, TN on May 21, 1970. He received his B.S. degree in electrical engineering from the University of Tennessee, Knoxville in 1992 and his M.S.E.E. from the Georgia Institute of Technology, Atlanta, GA, in 1993. He is presently working on his $\mathrm{Ph}$.D. degree also in electrical engineering at the Georgia Institute of Technology. His research work involves the numerical modeling of semiconductor devices with emphasis in the driftdiffusion and hydrodynamic simulation of photodetectors and avalanche photodiodes.
Kevin F. Brennan received the B.S. degree in physics from the Massachusetts Institute of Technology, Cambridge, in 1978, and the M.S. degree in physics and Ph.D. degree in electrical engineering from the University of Illinois, Urbana-Champaign, in 1984.

$\mathrm{He}$ is currently Institute Fellow and Professor, School of Electrical and Computer Engineering, Georgia Institute of Technology, Atlanta. His current research interests include the physics and modeling of semiconductor devices. Of particular interest are the physics and modeling of avalanche photodiodes, confined state ionization devices, high field effects in semiconductors, photoconductors, and high speed transistors.

Dr. Brennan was the recipient of a Presidential Young Investigator Award through the National Science Foundation.

Arlynn W. Smith received the B.S. degree in ceramic engineering from Alfred University, Hornell, NY, in 1984, the M.S. degree from the Georgia Institute of Technology, Atlanta, GA, in 1987, and the Ph.D. degree in electrical engineering from Georgia Tech. in 1992.

From 1992 to 1993, he held a post-doctoral fellowship at the Georgia Tech Research Institute, where he developed a three-dimensional simulation code for modeling the chemical vapor infiltration of ceramic matrix composites. From 1993 to 1995, he performed a post-doctoral fellowship with the microelectronics research center at Georgia Tech, where he developed a non-parabolic hydrodynamic simulation code for heterojunction acoustic charge transport, charge transport devices, and avalanche photodiodes. Currently, Dr. Smith is working for ITT Night Vision in Roanoke, VA developing advanced cathode structures for night vision applications. 

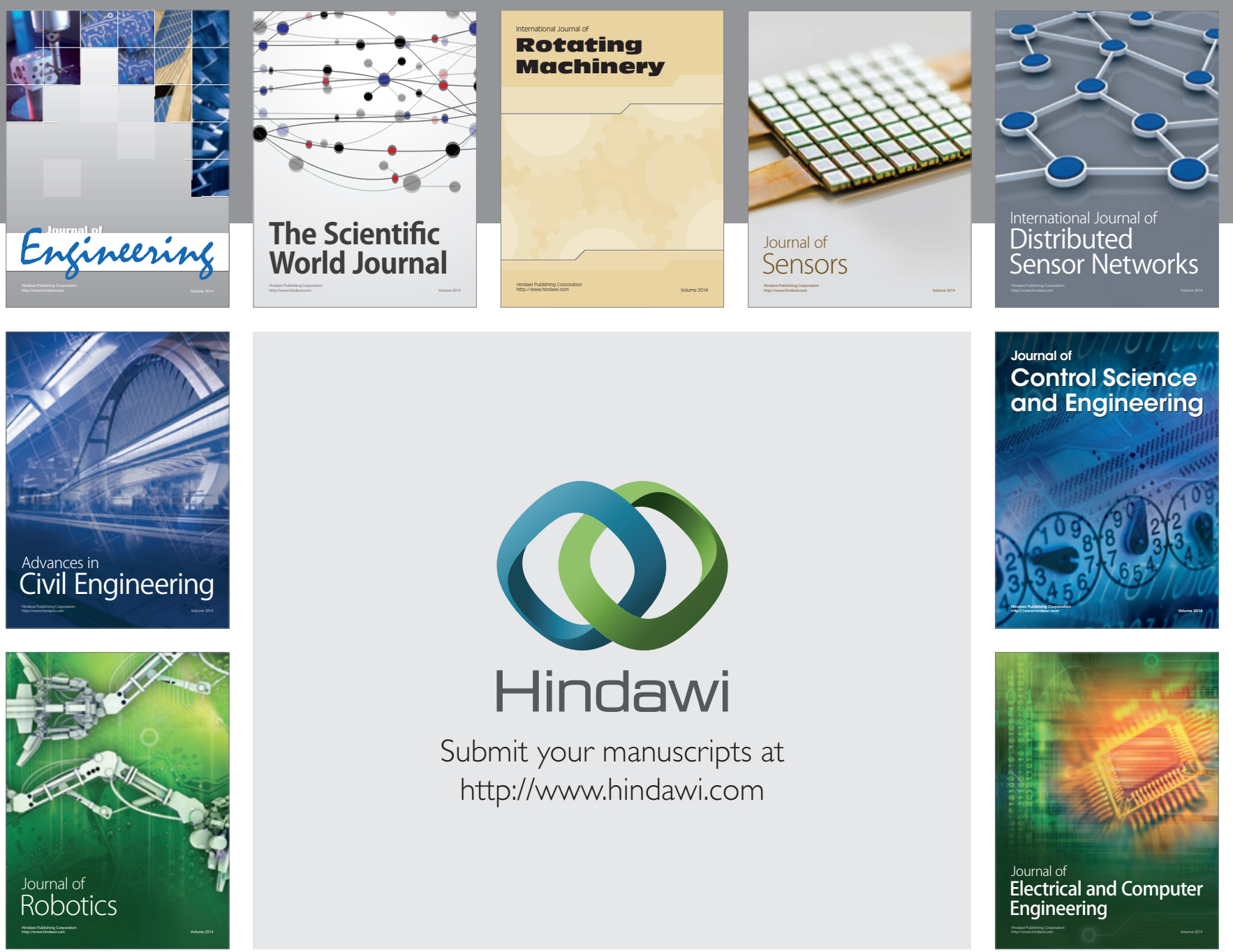

Submit your manuscripts at

http://www.hindawi.com
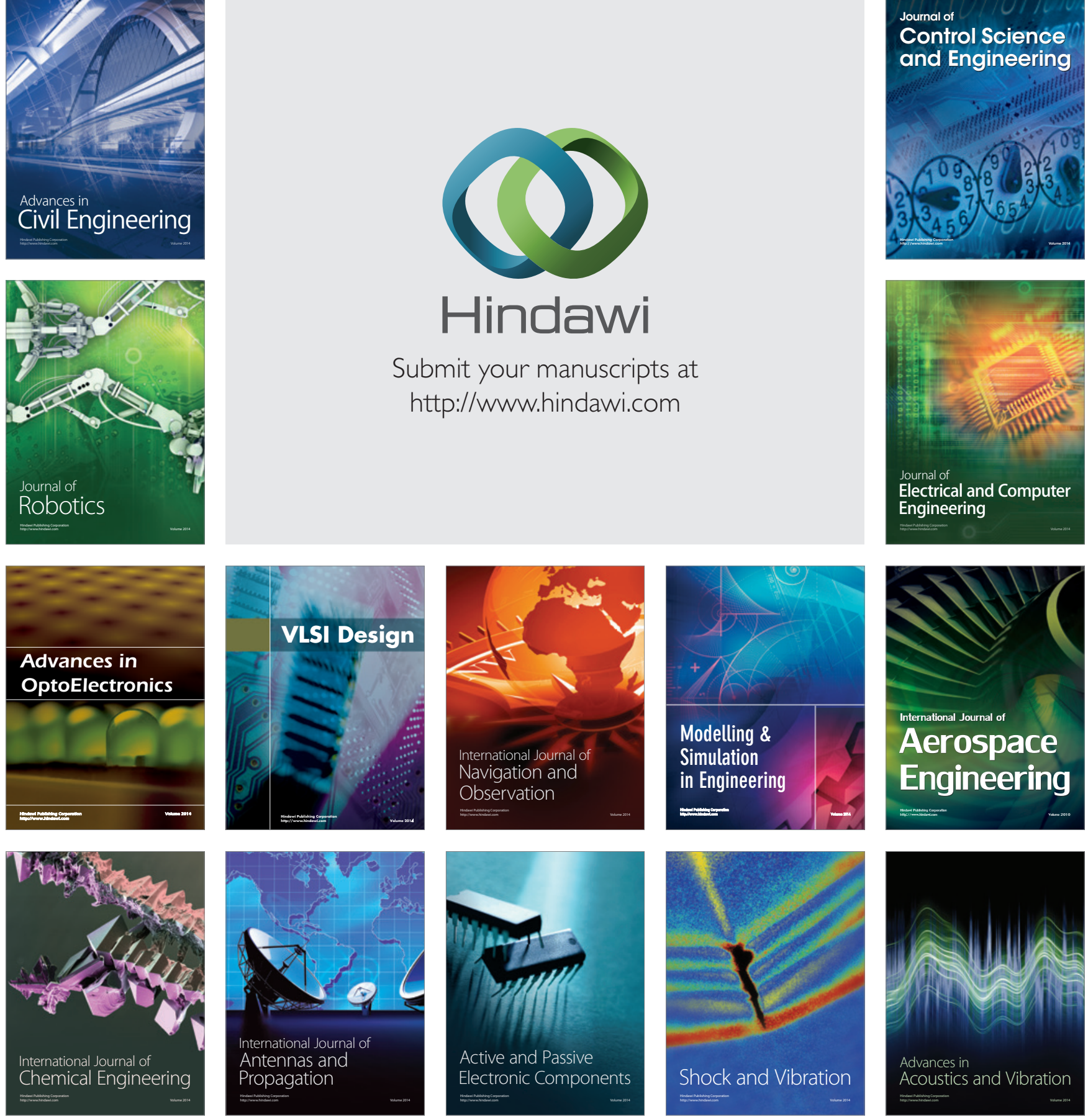